\title{
AZIMUTHAL STABILITY OF TRICHEL PULSES AND CATHODE DIRECTED STREAMERS
}

\section{O. Bolotov, B. Kadolin, S. Mankovskyi, V. Ostroushko, I. Pashchenko, G. Taran, L. Zavada National Science Center "Kharkov Institute of Physics and Technology”, Kharkiv, Ukraine E-mail: ostroushko-v@kipt.kharkov.ua}

The numerical simulations of negative corona discharge in Trichel pulse mode are carried out with the calculation of evolution of azimuthal perturbations. It is found the azimuthal instability with increment corresponding to avalanche development. This instability is suppressed at the nonlinear stage and does not lead to the process branching. The result is also applicable to the azimuthal instability of the cathode directed streamer, found earlier. The difference in their increments is followed from the difference in courses of the processess, which is discussed.

PACS: 52.80.Hc

\section{INTRODUCTION}

Negative corona discharge is widely used in plasmachemical techniques, in particular, in ozone synthesis. To intensify required reactions, it is worthy to use negative corona in Trichel pulse mode at relatively high voltage value but to keep discharge from turn to stationary mode, which destructs ozone through the gas heating.

In a discharge with a very large voltage, which realization requires rapid voltage application, during a few nanoseconds, a branching of the ionization process is observed, which can affect the gas heating rate, but which is not observed for the quasi-stationary voltage application, with a characteristic time of change, much larger than the time of Trichel pulse development up to the maximum value of total current. In order to find the possibility of branching realization, the numerical simulations of negative corona discharge in Trichel pulse mode are carried out.

The real possibilities of the modern computers allow the detailed simulations of the axially symmetric processes. For some three-dimensional processes, such as branching of streamers, the initial, linear stage of their development can also be simulated on a twodimensional mesh $[1,2]$. In the present paper, this approach is applied to the Trichtel pulses. Strictly speaking, the concepts of stability and instability are related to the perturbations of the stationary mode. It is near to the stationary one the intermediate stage of the cathodedirected streamer propagation when its head is already far removed from the anode, but has not yet approached the cathode. Any pulse process in general is unsteady, and the formal calculation of the development of its small perturbations may give characteristics somewhat corresponding to reality only when the characteristic time of development of those perturbations will be much less than the characteristic time of development of the main axially symmetric pulse.

\section{SIMULATION MODEL}

In the present numerical simulations, it is taken into account the drift and diffusion of electrons and ions and the processes of impact ionization, attachment, detachment, electron-ion and ion-ion recombination. The equations used are similar to those given in [3].

In conditions of the atmospheric pressure gas discharge, the electron and ion motion may be considered in the drift-diffusion approximation, and the field may be calculated as electrostatic. The time evolution of the particle densities and the field potential distribution may be determined with the equations

$$
\begin{gathered}
\partial_{\mathrm{t}} N_{e}-\operatorname{div}\left(D_{e} \nabla N_{e}+N_{e} \mu_{e} \vec{E}\right)= \\
=\left(v_{i}-v_{a}\right) N_{e}+v_{d} N_{n}-\beta_{e p} N_{e} N_{p}, \\
\partial_{\mathrm{t}} N_{p}-\operatorname{div}\left(D_{p} \nabla N_{p}-N_{p} \mu_{p} \vec{E}\right)= \\
=v_{i} N_{e}-\beta_{e p} N_{e} N_{p}-\beta_{n p} N_{n} N_{p}, \\
\partial_{\mathrm{t}} N_{n}-\operatorname{div}\left(D_{n} \nabla N_{n}+N_{n} \mu_{n} \vec{E}\right)= \\
=v_{a} N_{e}-v_{d} N_{n}-\beta_{n p} N_{n} N_{p}, \\
\nabla^{2} \Phi=-q \varepsilon_{0}^{-1}\left(N_{p}-N_{e}-N_{n}\right) .
\end{gathered}
$$

Here the indexes $e, p$, and $n$ indicates electrons, positive and negative ions, $\mu$ are relevant mobilities, $\beta$ are recombination coefficients, $v_{i}, v_{a}, v_{d}$ are frequencies of ionization, attachment, and detachment (numbers of the events per time caused by single electron or negative ion, respectively), $v_{i}=\alpha \mu_{e} E$, where $\alpha$ is ionization coefficient, $E=|\vec{E}|, \vec{E}=-\nabla \Phi, q$ is elementary charge, $\varepsilon_{0}$ is electric constant.

The calculations are carried out for the volume restricted with the ellipsoid of revolution having the focuses on the axis of revolution and with the hyperboloids of revolution having the same axis of revolution and the focuses. At the electrodes-hyperboloids it is imposed the conditions of absence of diffusion flow, absence of ion emission, and existence of electron emission from the cathode determined by positive ions flow,

$$
N_{e} \mu_{e} E=\gamma_{i} N_{p} \mu_{p} E,
$$

where $\gamma_{i}$ is the coefficient of ion-electron emission. At the boundary surface formed with ellipsoid, it is imposed the condition of absence of any charged particle flow to the surface or from the surface. To avoid the accumulation of the charged particles near this boundary it is assumed that in the elementary volumes nearest to this surface the charged particles have artificially large mobility. Potential is calculated in assumption of its fixed values at infinite hyperboloids-electrodes (one of them may be plane), and so, calculated field distribution corresponds to the case of infinite space between hyperboloids with charge in the volume bounded by ellipsoid. The calculations are carried out in the hyperboloid coordinates $(\sigma, \tau)$ connected with the cylindrical coordinates $(\rho, z)$ by the equalities 
$\rho=a\left[\left(\sigma^{2}-1\right)\left(1-\tau^{2}\right)\right]^{1 / 2}$ and $z=a \sigma \tau$, where $a$ is half of distance between focuses. To calculate the potential distribution the expansion in the terms of eigenfunctions with respect to the coordinate $\tau$ is made, and the obtained ordinary differential equation with respect to the coordinate $\sigma$ is solved with run method.

The evolution of the azimuthally inhomogeneous distribution is calculated in the linear approximation. For the particle densities and potential, it is taken

$$
N=N_{0}+N_{1} \cos (m \varphi), \Phi=\Phi_{0}+\Phi_{1} \cos (m \varphi),
$$

where $\varphi$ is azimutal angle (so that $x=\rho \cos \varphi$, $y=\rho \sin \varphi), m$ is natural number (the indexes epn here are not written). The sinuous streamer path may be connected with the development of the process at $m=1$, and the processes at $m \geq 2$, in particular, may describe the streamer bifurcation. It is worthy to write some equalities used in linear approximation: for the absolute value of field strength, $E=E_{0}+E_{1} \cos (m \varphi)$, where $E_{0}=\left|\vec{E}_{0}\right|, \quad E_{1}=\left(\vec{e}_{0}, \vec{E}_{1}\right), \vec{e}_{0}=\vec{E}_{0} / E_{0}$; for the perturbations (namely, for the corresponding factors at $\cos (m \varphi))$ of mobilities, $\mu_{1}=\mu^{(1)} E_{1}$, where $\mu^{(1)}$ is the derivative of the function $\mu(E)$; for the perturbation of ionization frequency, $v_{i 1}=\alpha_{0} \mu_{0} E_{1}+\alpha_{0} \mu_{1} E_{0}+\alpha_{1} \mu_{0} E_{0}$, where $\alpha_{1}=\alpha^{(1)} E_{1}$; for the perturbation of the quantity $\operatorname{div}(N \mu \vec{E})$, which has the form

$$
\operatorname{div}\left(N_{1} \mu_{0} \vec{E}_{0}+N_{0} \mu_{1} \vec{E}_{0}\right)+\left(\vec{E}_{1}, \nabla\left(N_{0} \mu_{0}\right)\right)+N_{0} \mu_{0} Q_{1},
$$

where $Q_{1}=q \varepsilon_{0}^{-1}\left(N_{p 1}-N_{e 1}-N_{n 1}\right)$; for the perturbation of summands connected with recombination, $\beta_{1} N_{0} N_{p 0}+\beta_{0} N_{1} N_{p 0}+\beta_{0} N_{0} N_{p 1}$. The equation for the perturbation of potential has the form $\nabla^{2} \Phi_{1}-m^{2} \rho^{-2} \Phi_{1}=-Q_{1}$. The diffusion coefficients are taken constant. With the approach to the symmetry axis the ratios of the perturbation and the quantity $\rho^{m}$ approaches the bounded values, so, it is worthy to remove the factor $\left(\sigma^{2}-1\right)^{m / 2}$ and to rewrite the equations for the coefficients at it. In particular, for the perturbation of potential the solution is searched in the form of the expansion $\Phi_{1}=\sum_{v} \Phi_{1 v}(\sigma) \mathrm{F}_{v}^{m}(\tau) \quad$ (with unknown $\left.\Phi_{1 v}(\sigma)\right)$ over the eigenvalues $v$ and the corresponding eigenfunctions $\mathrm{F}_{v}^{m}(\tau)$ of the Dirichlet problem for the Legendre equation,

$\partial_{\tau}\left[\left(1-\tau^{2}\right) \partial_{\tau} \mathrm{F}_{v}^{m}(\tau)\right]+\left[v(v+1)-m^{2}\left(1-\tau^{2}\right)^{-1}\right] \mathrm{F}_{v}^{m}(\tau)=0$.

Using the Poisson equation in hyperboloid coordinates one can obtain the equation

$$
\begin{gathered}
\partial_{\sigma}\left[\left(\sigma^{2}-1\right) \partial_{\sigma} \Phi_{1 v}(\sigma)\right]+ \\
+\left[-v(v+1)-m^{2}\left(\sigma^{2}-1\right)^{-1}\right] \Phi_{1 v}(\sigma)=-Q_{1 v}(\sigma),
\end{gathered}
$$

where the quantities $Q_{1 v}(\sigma)$ may be obtained from the expansion $Q_{1} a^{2}\left(\sigma^{2}-\tau^{2}\right)=\sum_{v} Q_{1 v}(\sigma) \mathrm{F}_{v}^{m}(\tau)$. Assumed that $\Phi_{1 v}(\sigma)=\bar{\Phi}_{1 v}(\sigma)\left(\sigma^{2}-1\right)^{m / 2}$, one can get the equation,

$$
\begin{aligned}
& \left(\sigma^{2}-1\right) \partial_{\sigma}^{2} \bar{\Phi}_{1 v}(\sigma)+2(m+1) \sigma \partial_{\sigma} \bar{\Phi}_{1 v}(\sigma)+ \\
& +[m(m+1)-v(v+1)] \bar{\Phi}_{1 v}(\sigma)=-\bar{Q}_{1 v}(\sigma),
\end{aligned}
$$

and the boundary condition at the symmetry axis,

$$
\begin{gathered}
\lim _{\sigma \rightarrow 1}\left\{2(m+1) \partial_{\sigma} \bar{\Phi}_{1 v}(\sigma)+\right. \\
\left.+[m(m+1)-v(v+1)] \bar{\Phi}_{1 v}(\sigma)+\bar{Q}_{1 v}(\sigma)\right\}=0 .
\end{gathered}
$$

As the initial distribution for the basic, axially symmetric mode, the uniform distribution with a number density of electrons and positive ions of $10^{3} \mathrm{~cm}^{-3}$ is taken, and for azimuthal harmonics (the amplitudes of which are normalized at each step), it is taken the distribution $\cos (m \varphi)$.

\section{SIMULATION RESULTS}

If the increment of a small azimuthal perturbation development is near (by the order of magnitude) to the inverse time of the main pulse development then the results of calculations of the perturbation evolution would depend to a large extent on the choice of the initial distribution. But it is revealed that the rate of azimuthal perturbations development significantly exceeds the rate of the main pulse development. In the Fig. 1, it is shown the typical time dependence for the total current $(I)$ and for the logarithmic derivatives of the maximum density in the axially symmetric mode $\left(\kappa_{0}\right)$ and in the azimuthal perturbation $(\kappa)$. It should be emphasized that for the different azimuthal harmonics (the calculations are carried out for $m=1 \ldots 6$ ) the increments are approximately equal. The value 1 on the ordinate axis corresponds to the following values: $10 \mathrm{~mA}$ for the total current, $10^{10} \mathrm{~s}^{-1}$ for the increment of the axiallysymmetric mode, $2.5 \cdot 10^{12} \mathrm{~s}^{-1}$ for the increment of the perturbation.

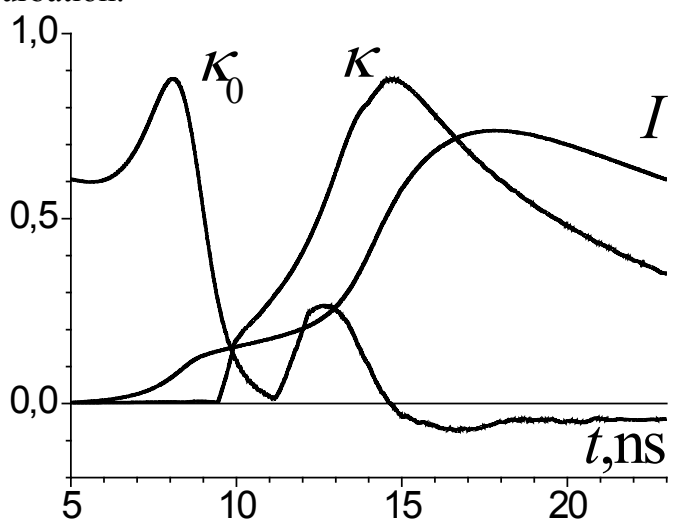

Fig. 1. Total current ( $I$ ) and time derivatives of maximum density logarithm, in axially-symmetric mode $\left(\kappa_{0}\right)$

and in azimuthal perturbation ( $\kappa)$; units in text

So, the instability of azimuthal perturbations with a large increment has been formally revealed, while in reality the branching of the process during the Trichel pulse is not observed. And it is noteworthy the fact that the obtained value of increment in order of magnitude corresponds to the characteristic frequency of ionization in a strong field. Therefore, it is natural to suppose that the obtained magnitude of the perturbation increase rate characterizes the linear stage of the electron avalanches development, up to the stage of the space charge field relaxation caused by conductivity. The ability of the avalanche to develop remains high at the stage of Trichel pulse dumping, but each avalanche, having been developed to the stage of field relaxation, reduces the rate 
of its development. This azimuthal instability of the Trichtel pulse mode does not lead to the ionization process branching. Some random heterogeneity of the structure, in particular, the advantage in the ion-electron emission coefficient of one areas of the cathode surface on others, leads to more intensive development of the ionization process in the corresponding direction, but the subsequent random perturbations complicate the details of this development almost imperceptibly.

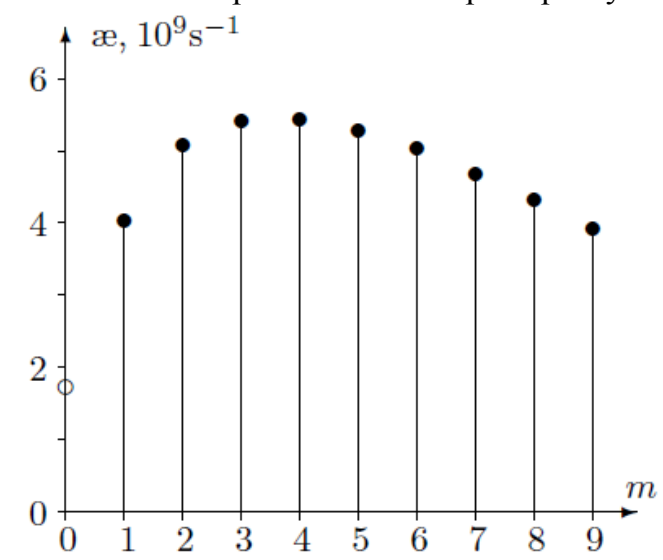

Fig. 2. Perturbation growth increments

for the azimuthal harmonics in cathode-directed streamer quasi-stationary propagation

In connection with the above, a question arises about the instability found in numerical simulation of the cathode-directed streamer $[1,2]$. There the development of azimuthally-inhomogeneous fluctuations was studied for a formally stationary (within the model) mode of a streamer propagation with constant velocity in an external field close to the homogeneous one. The increment of instability found there was much less than the ionization frequency and somewhat dependent on the harmonic number, as shown in the Fig. 2, corresponding to the Fig. 2 of the paper [2]. Here one should pay attention to the difference in the processes of the streamer propagation to cathode in positive corona, and Trichel pulse development in the negative corona. In the case of Trichel pulse, the electrons move in the direction of the ionized space propagation, and those ionization acts, due to which this propagation occurs, are performed by a significant part of electrons, so that the average number of successive acts of ionization performed by one of those electrons is relatively small. In the case of cathode directed streamer, the propagation of the ionized space takes place through an avalanche multiplication of electrons, the initial density of which in the air is $10^{8} \ldots 10^{9}$ times less than one characteristic of the streamer channel. The electrons move towards the streamer, becoming part of the streamer after performing about 30 acts of impact ionization $\left(9 \cdot \log _{2} 10 \approx 30\right)$. The first acts occur in a relatively weak field, where the time between successive acts of impact ionization performed by one electron is larger. As a result, the increment of perturbation density increase for cathode directed streamer is not 30, but approximately 100 times less than one for Trichel pulse, as it follows from the comparison of the Figs. 1 and 2. And the dependence of increment on the perturbation harmonic number in the Fig. 2 is related to the difference in the field strength of different harmonics at the distance from the streamer head near to its transverse dimension, for the same charge perturbation amplitude at the head. Whereas just on the head, in front of cathode directed streamer channel, the field strength of different harmonics for the same charge perturbation amplitude almost does not differ, but depends on the sizes related to the zone of intense ionization in front of the plasma space. Similarly, in negative corona, the field strength in the zone of intense ionization near the needle cathode during Trichel pulse development is almost the same for different harmonics, at the same amplitude of space charge perturbation.

It should be emphasized that the fact of realization of negative corona at the quasi-stationary voltage in the form of Trichel pulses, and not streamers, is closely related to the directions of the propagation of processes and the motion of electrons. The processes caused by ionization, developing faster in a strong field near the needle electrode, start up there and propagate to a plane electrode, whereas the electrons necessary for ionization move from cathode to anode, regardless of the shape of the electrodes. It is worthwhile to describe in more detail how the tendency to transverse localization of the process in streamer mode of positive corona and the absence of such tendency in Trichel pulse mode of negative corona is related to the directions of motion.

\section{TRANSVERSE LOCALIZATION}

For a positive streamer, which propagates from anode to cathode and is charged positively, transverse localization is a consequence of correlation of some parameters inherent to such a streamer.

First, the field strength level in the zone of intensive ionization in front of the streamer head is largely determined by the type of dependence of the ionization coefficient on the field strength value. Approximately, the dependence has the form $\alpha=\alpha_{0} \exp \left(-E_{\mathrm{a}} / E\right)$, where $E_{\mathrm{a}} \sim 200 \mathrm{kV} / \mathrm{cm}$. At $E<E_{\mathrm{a}}$ the dependence $\alpha$ on $E$ is very steep, and at $E \gg E_{\text {a }}$ it gradually goes to saturation. When the streamer propagates inside the gap at the given value of the potential of the streamer head relative to cathode, the value of the product $d_{\mathrm{s}} E_{\mathrm{m}}$ of the streamer transverse dimension $d_{\mathrm{s}}$ and the maximum intensity of the field strength $E_{\mathrm{m}}$ near the streamer head is approximately fixed. This assertion is connected with the fact that the potential, with respect to infinity, of the sphere with diameter $d_{\mathrm{s}}$ and the field strength $E_{\mathrm{m}}$ on its surface is equal to $d_{\mathrm{s}} E_{\mathrm{m}} / 2$. The streamer propagation velocity $v_{\mathrm{s}}$ is estimated by the relation $v_{\mathrm{s}} \sim d_{\mathrm{s}} v_{\mathrm{i}} \Lambda^{-1}$, where $v_{\mathrm{i}}$ is the characteristic frequency of impact ionization, $v_{\mathrm{i}} \sim \alpha \mu_{\mathrm{e}} E_{\mathrm{m}}, \alpha$ and $\mu_{\mathrm{e}}$ are characteristic values of the ionization coefficient and the electron mobility in the intensive ionization zone, $\Lambda$ is the number of ionization acts necessary to increase the electron density from the initial one to that which is characteristic for the streamer channel $(\Lambda \sim 25 \ldots 30)$. This assertion is based, in particular, on the assumption that the streamer propagation velocity is much greater than electron drift velocity, and intensive ionization, 
with frequency $v_{\mathrm{i}}$, begins at a distance $d_{\mathrm{s}}$ from the current position of the streamer head. Under these assumptions, after $\Lambda$ successive ionization acts with time intervals $1 / v_{\mathrm{i}}$, that is, in time $\Lambda / v_{\mathrm{i}}$, the forward part of the streamer channel propagates to a distance, which is slightly smaller than $d_{\mathrm{s}}$ (on the length of the electron drift during the time $\left.\Lambda / v_{\mathrm{i}}\right)$. As a result, $v_{\mathrm{s}} \sim d_{\mathrm{s}} E_{\mathrm{m}} \Lambda^{-1} \alpha \mu_{\mathrm{e}}$, and the streamer velocity is roughly proportional to the product $\alpha \mu_{\mathrm{e}}$. When the field strength increases, the electron mobility decreases, and the dependence of the product of ionization coefficient and electron mobility on the field strength has maximum at $E \sim E_{\mathrm{a}}$. That is, the streamer propagation velocity is maximal when $E_{\mathrm{m}} \sim E_{\mathrm{a}}$, and this value of $E_{\mathrm{m}}$ corresponds to some value $d_{\mathrm{s} 0}$ of the transverse streamer dimension $d_{\mathrm{s}}$, as the value of the product $d_{\mathrm{s}} E_{\mathrm{m}}$ is approximately fixed. If the streamer dimension $d_{\mathrm{s}}$ at the given value of head potential relative to cathode was considerably larger than $d_{\mathrm{s} 0}$, then from the streamer head, the new, smaller streamer, with $d_{\mathrm{s}} \sim d_{\mathrm{s} 0}$, would begin to propagate with a higher speed, whereas if the condition $d_{\mathrm{s}}<<d_{\mathrm{s} 0}$ was executed then the streamer would increase its transverse dimension and velocity until the condition $E_{\mathrm{m}} \sim E_{\mathrm{a}}$ is established.

Secondly, the electron density in the positive streamer channel is also, by the order of magnitude, determined by the values of the ionization coefficient and mobility of electrons at $E \sim E_{\mathrm{a}}$. Namely, through the avalanches multiplication the density of electrons increases, and therefore, the characteristic time $\tau_{\mathrm{r}}$ of the field relaxation, $\tau_{\mathrm{r}}=\sigma^{-1} \varepsilon_{0}$, where $\sigma$ is the conductivity, $\sigma=q N_{\mathrm{e}} \mu_{\mathrm{e}}$, decreases. When $\tau_{\mathrm{r}}$ becomes less than the characteristic time $1 / v_{\mathrm{i}}$ between two successive ionization acts carried out by one electron, the field after the last ionization act has time to weaken, and the next ionization act does not occur. Thus, the maximum electron density, attainable in an avalanche, is estimated by the ratio $N_{\mathrm{e}} \sim\left(q \mu_{\mathrm{e}}\right)^{-1} \varepsilon_{0} v_{\mathrm{i}}$, in which the value $v_{\mathrm{i}}$ and $\mu_{\mathrm{e}}$ should be taken for the field strength near to $E_{\mathrm{a}}$. For the atmospheric air, one gets $N_{\mathrm{e}} \sim\left(10^{14} \ldots 10^{15}\right) \mathrm{cm}^{-3}$.

Thirdly, the source of initial electrons for the propagation of avalanches in the atmospheric air is mainly ionization by photons emitted from such excited states of the nitrogen molecule, for which the photon energy exceeds the ionization energy of the oxygen molecule. The density of electrons obtained due to such ionization when the streamer propagates reaches the value $\left(10^{6} \ldots 10^{7}\right) \mathrm{cm}^{-3}$. The number of acts of impact ionization required to increase the density of electrons from this value to the value $\left(10^{14} \ldots 10^{15}\right) \mathrm{cm}^{-3}$ characteristic for the streamer channel is estimated to be $25 \ldots 30$ and it depends on the values of those densities logarithmically.

As a result, such a large number of successive acts of impact ionization in front of the streamer, coupled with the steep dependence $\alpha$ of $E$ when $E<<E_{\mathrm{a}}$, lead to a transversal localization of the streamer propagation process. For example, the difference of only $2.5 \%$ in the ionization coefficients for two close avalanche development paths after 30 acts of ionization gives approximately twice difference in the attained electron density, with the corresponding consequences for the prospect of streamer propagation in the corresponding direction. So, the transverse localization of propagation is an intrinsic feature of positive streamers.

The above considerations relate just to positive streamers, in which the development of avalanches begins at the points far from the streamer head, and propagates towards the head. On the contrary, in the ionization process, which propagates in the direction of the electron motion, the propagation starts from the volume with already attained high electron density. Then for the corresponding movement of the ionization front it is sufficient only one ionization act, and the small difference in the ionization coefficient for the different directions gives a small difference in the rate of the ionization front propagation for those directions. That is, it turns out that the negative streamers, directed to anode, cannot exist.

But the presented description of the ionization process propagation does not take into account the possibility of its branching, and refers to the case of the quasistationary voltage application and a slight excess of quasi-stationary voltage over the relevant streamer mode threshold. The discharge is realized at such voltage value in the case when voltage is increased slowly and the intensive ionization process development occurs just after the voltage exceeds the threshold. If the voltage is applied for the time of the order of nanoseconds, then the process goes on just in the field corresponding to the instantaneous value of the applied voltage, which can significantly exceed the threshold. And then the points, in which $E \sim E_{\text {a }}$, can be located far from the current position of the negative streamer head. And before the streamer head approaches those points through ionization, along with the displacement of electrons from the former location of the head, from those points it has time to develop an avalanche, the backward end of which largely has the properties of the streamer channel. And for the old head, to become the part of that channel it is sufficient to reach it. In addition, from that channel, it may begin the process of the positive streamer propagation, directed to the head. In any case, the connection of the old head with the high conductive plasma corresponds to the formation of a continuous channel of the streamer, which has already propagated further. But there may be a lot of the points, from which the development of avalanches begins, and then, with a high probability, the streamer branching may occur.

The positive streamer, directed to the cathode, also can propagate at the voltage value significantly greater than the streamer mode threshold in the case of the quasi-stationary voltage application. And then the development of avalanches starts from many points, more distant from the head, than it is in the case of voltage close to the threshold. And after the avalanche coming to the head, a few remnants of their traces can turn into 
channels of the branched positive streamer. However, positive streamers can exist and branch out also at a voltage close to the threshold.

\section{CONCLUSIONS}

In the numerical simulations of negative corona discharge in Trichel pulse mode with the calculation of the evolution of azimuthal perturbations, it is found the azimuthal instability with the value of increment corresponding to electron avalanche multiplication through the impact ionization. This formally obtained instability does not lead to the branching of the process in reality, because it is suppressed at the nonlinear stage of the avalanche development. It becomes obviously that the azimuthal instability of the cathode directed streamer propagation in positive corona discharge obtained earlier has the same nature. The difference of these instabilities in the increment values is connected with the directions of the electron motion and the process propagation: in the negative corona the direction is the same, whereas in the positive corona the directions are opposite. It is discussed, how the difference in the process courses is connected with the absence of the streamer mode in the negative corona at the quasi-stationary voltage application.

\section{REFERENCES}

1. O. Bolotov, B. Kadolin, S. Mankovskyi, et al. Quasistationary streamer propagation // Problems of Atomic Science and Technology. Series "Plasma Electronics and New Methods of Acceleration”. 2015, № 4, p. 185-188.

2. O. Bolotov, B. Kadolin, S. Mankovskyi, et al. Numerical simulations of quasi-stationary streamer propagation // Problems of Atomic Science and Technology. Series "Nuclear Physics Investigations”. 2016, № 5, p. 121-125.

3. O. Bolotov, V. Golota, B. Kadolin, et al. Development of azimuthally not uniform processes in corona discharge in axially symmetric gap // Problems of Atomic Science and Technology. Series "Plasma Electronics and New Methods of Acceleration". 2013, № 4, p. 161-165.

Article received 02.06.2019

\section{АЗИМУТАЛЬНАЯ УСТОЙЧИВОСТЬ ИМПУЛЬСОВ ТРИЧЕЛА И НАПРАВЛЕННЫХ К КАТОДУ СТРИМЕРОВ}

О. Болотов, Б. Кадолин, С. Маньковский, В. Остроушко, И. Пащенко, Г. Таран, Л. Завада

Выполнено численное моделирование отрицательной короны в режиме импульсов Тричела с расчетом эволюции азимутальных возмущений. Выявлена азимутальная неустойчивость с инкрементом, соответствующим развитию лавины. Эта неустойчивость подавляется на нелинейной стадии и не ведет к ветвлению процесса. Результат также применим к выявленной ранее азимутальной неустойчивости направленного к катоду стримера. Разница в их инкрементах следует из разницы в ходе процессов, которые обсуждаются.

\section{АЗИМУТАЛЬНА СТІЙКІСТЬ ІМПУЛЬСІВ ТРИЧЕЛА ТА СПРЯМОВАНИХ ДО КАТОДА СТРИМЕРІВ}

\section{О. Болотов, Б. Кадолін, С. Маньковський, В. Остроущко, І. Пащенко, Г. Таран, Л. Завада}

Виконане числове моделювання негативної корони в режимі імпульсів Тричела з розрахунком еволюції азимутальних збурень. Виявлено азимутальну нестійкість з інкрементом, відповідним розвитку лавини. Ця нестійкість пригнічується на нелінійній стадії і не веде до галуження процесу. Результат також застосовується до виявленої раніше азимутальної нестійкості спрямованого до катода стримера. Різниця в їхніх інкрементах випливає з різниці в ході процесів, які обговорюються. 